\title{
Processos pontuais no modelo de Guiol-Machado-Schinazi de sobrevivência de espécies
}

\author{
Maicon Aparecido Pinheiro
}

\author{
DissertaÇÃo/TESE APRESENTADA \\ $\mathrm{AO}$ \\ Instituto DE MATEMÁticA E EstatísticA \\ DA \\ Universidade De SÃo Paulo \\ PARA \\ OBTENÇÃO DO TÍTULO \\ $\mathrm{DE}$ \\ Mestre em CiênCIAS
}

Programa: Estatística

Orientador: Prof. Dr. Luiz Renato Gonçalves Fontes

Durante o desenvolvimento deste trabalho o autor recebeu auxílio financeiro da CAPES e CNPq

São Paulo, setembro de 2015 


\section{Processos pontuais no modelo de Guiol-Machado-Schinazi de sobrevivência de espécies}

Esta versão da dissertação contém as correções e alterações sugeridas pela Comissão Julgadora durante a defesa da versão original do trabalho, realizada em 13/07/2015. Uma cópia da versão original está disponível no Instituto de Matemática e Estatística da Universidade de São Paulo.

Comissão Julgadora:

- Prof. Dr. Luiz Renato Gonçalves Fontes (orientador) - IME/USP

- Prof. Dr. Fábio Prates Machado - IME/USP

- Prof. Dr. Cristian Favio Coletti - CMCC/UFABC 


\section{Agradecimentos}

Inicialmente, agradeço de forma geral a todos os meus amigos, que tornaram tudo mais simples e divertido. Ademais, manifesto de modo especial minha grande gratidão:

À minha família, que me deram apoio imensurável em todas as escolhas já feitas e trajetórias já traçadas: pessoas a quem dedico todo o meu respeito e carinho.

Ao Professor Luiz Renato Gonçalves Fontes, que esteve sempre à disposição e com uma enorme paciência para ensinar: alguém a quem devo muito de minha inicial autonomia acadêmica.

E, acima de tudo, à Deus! 


\section{Resumo}

PINHEIRO, M. A. Processos pontuais no modelo de Guiol-Machado-Schinazi de sobrevivência de espécies. 2015. 37 f. Dissertação (Mestrado) - Instituto de Matemática e Estatística, Universidade de São Paulo, São Paulo, 2015.

Recentemente, Guiol, Machado e Schinazi propuseram um modelo estocástico para a evolução de espécies. Nesse modelo, as intensidades de nascimentos de novas espécies e de ocorrências de extinções são invariantes ao longo do tempo. Ademais, no instante de nascimento de uma nova espécie, a mesma é rotulada com um número aleatório gerado de uma distribuição absolutamente contínua. Toda vez que ocorre uma extinção, apenas uma espécie morre - a com o menor número vinculado. Quando a intensidade com que surgem novas espécies é maior que a com que ocorrem extinções, existe um valor crítico $f_{c}$ tal que todas as espécies rotuladas com números menores que $f_{c}$ morrerão quase certamente depois de um tempo aleatório finito, e as rotuladas com números maiores que $f_{c}$ terão probabilidades positivas de se tornarem perpétuas. No entanto, espécies menos aptas continuam a aparecer durante o processo evolutivo e não há a garantia do surgimento de uma espécie imortal. Consideramos um caso particular do modelo de Guiol, Machado e Schinazi e abordamos estes dois últimos pontos. Caracterizamos o processo pontual limite vinculado às espécies na fase subcrítica do modelo e discorremos sobre a existência de espécies imortais.

Palavras-chave: Processos Pontuais, Processos de Markov, Evolução. 


\section{Abstract}

PINHEIRO, M. A. Point processes in the Guiol-Machado-Schinazi species survival model. 2015. 37 f. Dissertação (Mestrado) - Instituto de Matemática e Estatística, Universidade de São Paulo, São Paulo, 2015.

Recently, Guiol, Machado and Schinazi proposed a stochastic model for species evolution. In this model, births and deaths of species occur with intensities invariant over time. Moreover, at the time of birth of a new species, it is labeled with a random number sampled from an absolutely continuous distribution. Each time there is an extinction event, exactly one existing species disappears: that with the smallest number. When the birth rate is greater than the extinction rate, there is a critical value $f_{c}$ such that all species that come with number less than $f_{c}$ will almost certainly die after a finite random time, and those with numbers higher than $f_{c}$ survive forever with positive probability. However, less suitable species continue to appear during the evolutionary process and there is no guarantee the emergence of an immortal species. We consider a particular case of Guiol, Machado and Schinazi model and approach these last two points. We characterize the limit point process linked to species in the subcritical phase of the model and discuss the existence of immortal species.

Keywords: Point Processes, Markov Processes, Evolution. 


\section{Sumário}

1 Introdução $\quad \mathbf{1}$

1.1 Organização do trabalho . . . . . . . . . . . . . . . . . . . . . . 1

2 Processo pontual limite na fase subcrítica do modelo GMS $\quad 3$

2.1 Descrição do modelo . . . . . . . . . . . . . . . . . . . . 3

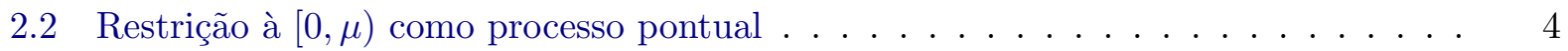

2.3 Variáveis de ocupação como processo markoviano de saltos . . . . . . . . . . . . 4

2.3.1 Propriedades da medida invariante para $k=1,2 \ldots \ldots \ldots \ldots$

2.4 Convergência em distribuição de $\mathcal{X}_{t}^{\prime} \ldots \ldots \ldots \ldots \ldots \ldots \ldots$

2.5 Propriedades da medida limite . . . . . . . . . . . . . . . . . . 14

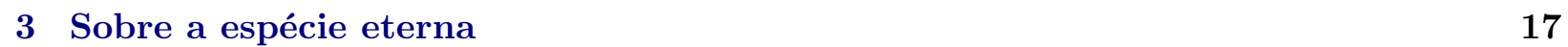

3.1 Infinitas espécies eternas . . . . . . . . . . . . . . . 17

3.2 Processo pontual de surgimento de espécies eternas . . . . . . . . . . . . . . . 19

4 Conclusões $\quad 25$

$\begin{array}{ll}\text { Referências Bibliográficas } & \mathbf{2 7}\end{array}$ 


\section{Capítulo 1}

\section{Introdução}

Em 2013, Guiol, Machado e Schinazi (Guiol et al., 2011), (Guiol et al., 2013) propuseram um modelo estocástico para a evolução de espécies. Nesse modelo, novas espécies surgem com taxa $\lambda$ e, com taxa $\mu$, ocorre um evento de extinção, em que uma espécie existente é extinta, segundo as seguintes regras. No instante do nascimento de uma nova espécie, é associada a ela um número aleatório gerado de uma dada distribuição F absolutamente contínua - tal número é interpretado como a propensão dessa espécie à sobrevivência e é chamado de aptidão. Cada vez que ocorre um evento de extinção, apenas uma espécie existente desaparece, qual seja, aquela com a menor aptidão. Essas aptidões são independentes uma das outras e de todo o resto do processo. Assim como em (Khouri, 2013), chamaremos esse modelo de GMS.

Em (Guiol et al., 2013), mostra-se que quando $\lambda>\mu$ existe um valor crítico determinístico $f_{c}$ não-trivial tal que todas as espécies que surgem com aptidões menores que $f_{c}$ desaparecem quase certamente depois de um tempo aleatório, e aquelas com aptidões maiores que $f_{c}$ sobrevivem para sempre com probabilidade positiva.

Sem perda de generalidade, nessa dissertação, consideramos o caso particular do modelo GMS em que $\mathrm{F}$ é a função distribuição de uma variável aleatória uniforme no intervalo [0,1]. Pelo fato de F ser absolutamente contínua, cada espécie é identificada por sua aptidão. Logo, para um instante de tempo $t$, as espécies vivas nesse instante podem ser vistas como um conjunto de pontos entre zero e um, isto é, uma realização de um processo pontual no intervalo [0,1]. Nosso primeiro interesse nesse trabalho é estudar o processo pontual vinculado às espécies vivas com aptidões no intervalo $\left[0, f_{c}\right)$ quando $t \rightarrow \infty$. O objetivo é entender a distribuição assintótica das espécies na fase subcrítica do modelo GMS.

Em um segundo momento, nos preocupamos com a existência de uma espécie que sobreviverá para sempre. Mostraremos que, eventualmente, surgirão várias espécies imortais. Como mencionado acima, isto só pode ocorrer para aptidões acima de $f_{c}$. Ademais, apresentamos estimativas para a taxa de decaimento (exponencial) da cauda da distribuição do tempo de surgimento da primeira espécie imortal a partir da condição inicial vazia.

\subsection{Organização do trabalho}

Organizamos o trabalho da seguinte forma. No próximo capítulo, Capítulo 2, discorremos sobre o comportamento assintótico do processo pontual de aptidões abaixo de $f_{c}$ em dado tempo $t$ quando o tempo diverge. No Capítulo 3, sobre a existência das espécies imortais e a probabilidade de que as mesmas apareçam conforme decorre o processo evolutivo. Por fim, no Capítulo 4, nossas conclusões. 


\section{Capítulo 2}

\section{Processo pontual limite na fase subcrítica do modelo GMS}

\subsection{Descrição do modelo}

Nessa seção, apresentamos uma construção para o modelo GMS. Para tal, sejam $\mathcal{N}$ e $\mathcal{M}$ dois processos de Poisson independentes, o primeiro com taxa $1 \mathrm{em}(0, \infty) \times(0,1)$ e o segundo com taxa $\mu$ em $(0, \infty) \times\{0\}$. Denotemos por $(\Omega, \mathcal{F}, \mathbb{P})$ o espaço de probabilidade subjacente. Definamos

$$
\mathcal{P}=\mathcal{M} \cup \mathcal{N} .
$$

Seja $\mathcal{X}_{0} \in \mathcal{B}((0,1))$ um boreliano do intervalo $(0,1)$. Dados $\mathcal{X}_{0}$ e $\mathcal{P}$, definamos $\mathcal{X}_{t}, t \geq 0$, da seguinte maneira:

Seja $T_{0}=0$ e definamos

$$
T_{1}=\inf \{t>0: \mathcal{P} \cap((0, t] \times[0,1)) \neq \emptyset\},
$$

que corresponde ao primeiro tempo $t$ em que ocorre uma marca em $\mathcal{P}$. Note que $T_{1} \sim \exp (1+\mu)$, isto é, $T_{1}$ é uma variável aleatória exponencialmente distribuída com parâmetro $1+\mu$. Daí, para todo $t \in\left(0, T_{1}\right)$, seja $\mathcal{X}_{t}=\mathcal{X}_{0}$.

Seja $X_{1} \in[0,1)$ tal que $\left(T_{1}, X_{1}\right) \in \mathcal{P}$. $\left(X_{1}\right.$ está definida quase certamente).

- Se $\left(T_{1}, X_{1}\right) \in \mathcal{N}$, então $\mathcal{X}_{T_{1}}=\mathcal{X}_{0} \cup\left\{X_{1}\right\}$, e

- Se $\left(T_{1}, X_{1}\right) \in \mathcal{M}$, então $\mathcal{X}_{T_{1}}=\mathcal{X}_{0} \backslash\left\{\inf \mathcal{X}_{0}\right\}$,

com a convenção de que $\{\inf \emptyset\}=\emptyset$.

Em palavras, a primeira situação representa o nascimento de uma nova espécie. A segunda, em direção oposta, indica a extinção da menos apta à sobrevivência. Note que até aqui temos o processo $\mathcal{X}_{t}$ construído até o tempo $T_{1}$.

Para $n \geq 2$, suponhamos definidos $T_{1}, \ldots, T_{n-1}$ e $\mathcal{X}_{t}$, para $t \leq T_{n-1}$. Então, sejam

$$
T_{n}=\inf \left\{t>T_{n-1}: \mathcal{P} \cap\left(\left(T_{n-1}, t\right] \times[0,1)\right) \neq \emptyset\right\}
$$

e $X_{n} \in[0,1)$ tal que $\left(T_{n}, X_{n}\right) \in \mathcal{P}$. Notemos que $T_{n}-T_{n-1} \sim \exp (1+\mu)$. Para $t \in\left(T_{n-1}, T_{n}\right)$, façamos $\mathcal{X}_{t}=\mathcal{X}_{T_{n-1}}$ e

- Se $\left(T_{n}, X_{n}\right) \in \mathcal{N}$, então $\mathcal{X}_{T_{n}}=\mathcal{X}_{T_{n-1}} \cup\left\{X_{n}\right\}$, 
- Se $\left(T_{n}, X_{n}\right) \in \mathcal{M}$, então $\mathcal{X}_{T_{n}}=\mathcal{X}_{T_{n-1}} \backslash\left\{\inf \mathcal{X}_{T_{n-1}}\right\}$.

Assim, por indução, podemos construir processo $\mathcal{X}_{t}$, que representa o processo das aptidões a partir de um dado boreliano $\mathcal{X}_{0}$ do intervalo $(0,1)$ como condição inicial. Notemos que $\mathcal{X}_{t}$ está bem definido para todo $t \geq 0$, já que $T_{n} \nearrow \infty$ quando $n \nearrow \infty$ quase certamente.

A construção dada acima refere-se ao caso particular do modelo GMS (Guiol et al., 2013) em que os nascimentos ocorrem com taxa 1 e as aptidões são geradas de uma distribuição uniforme no intervalo $[0,1]$.

\subsection{Restrição à $[0, \mu)$ como processo pontual}

Nesta seção, suporemos que $\mu<1$ e estudaremos o processo $\mathcal{X}_{t}^{\prime}:=\mathcal{X}_{t} \cap(0, \mu)$.

Notemos que pelas propriedades de independência de processos de Poisson, podemos construir $\mathcal{X}_{t}^{\prime}$ como $\mathcal{X}_{t}$, apenas substituindo $\mathcal{X}_{0}$ por $\mathcal{X}_{0}^{\prime}$ e $\mathcal{N}$ por $\mathcal{N}^{\prime}=\mathcal{N} \cap((0, \infty) \times(0, \mu))$ (que pode ser descrito como um processo de Poisson com taxa $1 \mathrm{em}(0, \infty) \times(0, \mu))$.

Vamos ademais supor que $\mathcal{X}_{0}^{\prime}$ é um subconjunto localmente finito de $[0, \mu)$, isto é, $\left|\mathcal{X}_{0}^{\prime} \cap[0, b]\right|<\infty$ para todo $b<\mu$. Notemos que esta propriedade se estende quase certamente para $\mathcal{X}_{t}^{\prime}, t \geq 0$, isto é, quase certamente

$$
\left|\mathcal{X}_{t}^{\prime} \cap[0, b]\right|<\infty, t \geq 0 .
$$

Em palavras, para cada $t \geq 0, \mathcal{X}_{t}^{\prime}$ é um processo pontual simples em $[0, \mu)$ - vide ((Daley e Vere-Jones , 2008), capítulo 9, seção 1 , Definição 9.1.VI, página 7). Podemos então ver $\left(\mathcal{X}_{t}^{\prime}\right)_{t \geq 0}$ em $[0, \mu)$ como um processo pontual dinâmico.

No restante deste capítulo vamos estabelecer o comportamento assintótico (da distribuição) de $\mathcal{X}_{t}^{\prime}$. Para tanto, vamos explorar dois aspectos da distribuição de $\mathcal{X}_{t}^{\prime}$ : as variáveis de ocupação e a função distribuição associada à $\mathcal{X}_{t}^{\prime}$.

\subsection{Variáveis de ocupação como processo markoviano de saltos}

Nessa seção, associamos ao modelo GMS um processo Markoviano de saltos construído a partir das chamadas variáveis de ocupação. Esse processo será extremamente importante para o estudo do comportamento assintótico de $\mathcal{X}_{t}^{\prime}$.

Vamos fixar $a \in(0, \mu)$ e considerar $\mathcal{X}_{t}^{\prime \prime}=\mathcal{X}_{t}^{\prime} \cap[0, \mu-a]$ o conjunto das aptidões menores que $\mu-a$ atreladas à espécies vivas no tempo $t$. Ademais, para $k \in \mathbb{N}^{*}$ fixo, seja $\mathrm{P}_{k}=\left\{x_{n}: n=0, \ldots, k\right\}$ a partição do intervalo $[0, \mu-a)$ onde $x_{n}=(n \mu-n a) / k \mathrm{e}$

$$
N_{t}^{i}=\left|\mathcal{X}_{t}^{\prime \prime} \cap\left[x_{i-1}, x_{i}\right)\right|
$$

o número de espécies vivas no instante $t$ com aptidões abaixo de $\mu-a$ e entre $x_{i-1}$ (inclusive) e $x_{i}$, $i=1, \ldots, k$.

Definição 2.3.1 (Variáveis de Ocupação). Dados $a \in(0, \mu)$ e $k \in \mathbb{N}^{*}$, para cada $t \geq 0$, as variáveis aleatórias $\left\{N_{t}^{i}: i=1, \ldots, k\right\}$ constituem o que chamamos de variáveis de ocupação no tempo $t$ da partição em $k$ subintervalos de $[0, \mu-a)$. 
Agora, para cada $t \geq 0$, seja $N_{t}=\left(N_{t}^{1}, N_{t}^{2}, \ldots, N_{t}^{k}\right)$. Das propriedades bem conhecidas de falta de memória dos processos de Poisson segue a propriedade de Markov e homogeneidade para $N_{t}$. Isto é, dados $0 \leq t_{0}<t_{1}<\ldots<t_{l}$,

$$
\mathbb{P}\left(N_{t_{l}}=\boldsymbol{n}_{l} \mid N_{t_{1}}=\boldsymbol{n}_{1}, \ldots N_{t_{l-1}}=\boldsymbol{n}_{l-1}\right)=\mathbb{P}\left(N_{t_{l}-t_{l-1}}=\boldsymbol{n}_{l} \mid N_{0}=\boldsymbol{n}_{l-1}\right),
$$

com $\boldsymbol{n}_{i}=\left(n_{i}^{1}, \ldots, n_{i}^{k}\right), n_{i}^{j} \in \mathbb{N}$. Além disso, podemos verificar sem maiores dificuldades que $N_{t}$ é um processo Markoviano de saltos homogêneo em $\mathbb{N}^{k}$ com as taxas de salto:

$$
\lambda\left(\boldsymbol{n} \rightarrow \boldsymbol{n}+\mathbf{1}_{j}\right)=\frac{\mu-a}{k}, \quad \text { para todo } j \in\{1, \ldots, k\} \text { e } \boldsymbol{n} \in \mathbb{N}^{k},
$$

e se $\boldsymbol{n}=\left(0, \ldots, 0, n^{j}, \ldots, n^{k}\right)$, onde $n^{j} \geq 1$ e $\left(n^{j+1}, \ldots, n^{k}\right) \in \mathbb{N}^{k-j}$, então

$$
\lambda\left(\boldsymbol{n} \rightarrow \boldsymbol{n}-\mathbf{1}_{j}\right)=\mu \quad \text { para todo } j \in\{1, \ldots, k\},
$$

onde $\mathbf{1}_{j}=(0, \ldots, 1, \ldots, 0) \in \mathbb{N}^{k}$ é o vetor com o valor 1 na $j$-ésima coordenada e 0 nas demais.

As transições acima são as únicas existentes. Note que fazendo

$$
S_{t}=N_{t}^{1}+\ldots+N_{t}^{k}
$$

temos que $\left(S_{t}\right)_{t \geq 0}$ é um processo de nascimento e morte em $\mathbb{N}$ com taxas de transição

$$
\begin{array}{ll}
\lambda(n \rightarrow n+1)=\mu-a, & \text { se } n \geq 0, \\
\lambda(n \rightarrow n-1)=\mu, & \text { se } n \geq 1 .
\end{array}
$$

Vamos estabelecer que $\left(N_{t}\right)_{t \geq 0}$ é irredutível e recorrente positivo, e logo tem uma única medida invariante.

Proposição 2.3.1. O processo $\left(N_{t}\right)_{t \geq 0}$ é irredutível.

Demonstração. Basta notar que todos os estados de $\mathbb{N}^{k}$ atingem e são atingidos por $\mathbf{0}=(0, \ldots, 0)$. Seja $\boldsymbol{n} \in \mathbb{N}^{k}$ tal que $n^{i} \geq 1$ para algum $i$ e suponha que $N_{0}=\boldsymbol{n}$. Considere o evento

$$
A=\{\mathcal{N} \cap[0,1] \times[0,1]=\emptyset\} \cap\{|\mathcal{M} \cap[0,1] \times\{0\}| \geq s\}
$$

onde $s=n^{1}+\ldots+n^{k}$. É claro que $\mathbb{P}(A)>0$ e, em $A, N_{1}=\mathbf{0}$. Temos então que $\boldsymbol{n}$ atinge $\mathbf{0}$ com probabilidade positiva. Agora, suponha que $N_{0}=\mathbf{0}$ e consideremos o evento

$$
B=\{\mathcal{M} \cap[0,1] \times\{0\}=\emptyset\} \cap\left\{\bigcap_{i=1}^{k}\left\{\left|\mathcal{N} \cap[0,1] \times\left[x_{i-1}, x_{i}\right)\right|=n^{i}\right\}\right\} .
$$

É claro que $\mathbb{P}(B)>0$ e que $N_{1}=\boldsymbol{n}$ em $B$. Logo, 0 atinge $\boldsymbol{n}$ com probabilidade positiva e temos que $\left(N_{t}\right)_{t \geq 0}$ é irredutível.

Proposição 2.3.2. O processo $\left(N_{t}\right)_{t \geq 0}$ é recorrente positivo.

Demonstração. É simplesmente mostrar que

$$
\mathbb{E}_{0}\left(\tau_{0}\right)<\infty
$$


onde $\tau_{0}=\inf \left\{t \geq \sigma_{0}: N_{t}=\mathbf{0}\right\}, \sigma_{0}=\inf \left\{t \geq 0: N_{t} \neq 0\right\}$ e $\mathbb{E}_{0}(\cdot)=\mathbb{E}\left(\cdot \mid N_{0}=\mathbf{0}\right)$. Note que

$$
\tau_{0}=\inf \left\{t>T_{1}: S_{t}=0\right\} .
$$

Da teoria de Processos de Nascimento e Morte, sabe-se que $\mathbb{E}_{0}\left(\tau_{0}\right)<\infty$ - vide (Durret, 2012), Capítulo 4, Exemplo 4.18, por exemplo.

Logo, temos para cada escolha de $k \geq 1$ e $x_{1}, \ldots, x_{k},\left(N_{t}\right)_{t \geq 0}$ tem uma única medida invariante.

Vamos a seguir estudar $\left(N_{t}\right)_{t \geq 0}$ no caso em que $k=2$. Este estudo fornecerá resultados a serem utilizados na sequência. Para tal, será necessário também o estudo para $k=1$.

\subsubsection{Propriedades da medida invariante para $k=1,2$}

- Caso $k=1$

Aqui, basta notar que $\left(N_{t}\right)_{t \geq 0}$ é equivalente a $\left(S_{t}\right)_{t \geq 0}$.

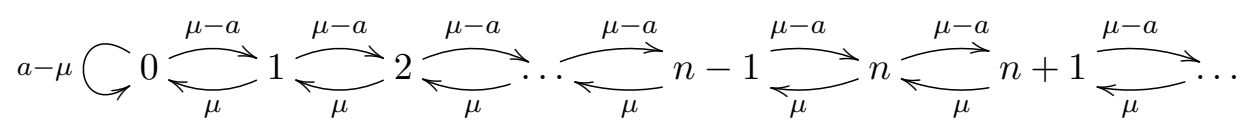

Figura 2.1: Representação pictorial dos estados e taxas para a cadeia $\left(N_{t}\right)_{t \geq 0}$.

A partir daí, obtemos o seguinte resultado, que é bem conhecido e está na literatura elementar sobre processos de nascimentos e morte.

Proposição 2.3.3. A medida invariante $\pi_{1}$ associada à cadeia $\left(N_{t}\right)_{t \geq 0}$ é tal que

$$
\pi_{1}(n)=\frac{a}{\mu}\left(1-\frac{a}{\mu}\right)^{n}, \quad \text { para } n \geq 0
$$

Demonstração. As equações de equilíbrio detalhado são dadas por

$$
\mu \pi_{1}(n)=(\mu-a) \pi_{1}(n-1) \quad \text { para } n \geq 1 .
$$

Ao utilizar a relação acima de modo iterativo, segue que

$$
\pi_{1}(n)=\left(1-\frac{a}{\mu}\right)^{n} \pi_{1}(0)
$$

Como $\pi_{1}$ é uma medida de probabilidade, devemos ter

$$
\sum_{n=0}^{\infty} \pi_{1}(n)=\sum_{n=0}^{\infty}\left(1-\frac{a}{\mu}\right)^{n} \pi_{1}(0)=\frac{\pi_{1}(0)}{a / \mu}=1 .
$$

Assim, a partir de $\pi_{1}(0)=a / \mu$ e da equação 2.17, segue o resultado. 
- Caso $k=2$

Nesse caso, o processo $\left(N_{t}\right)_{t \geq 0}$ toma valores em $\mathbb{N}^{2}$. Seja $\boldsymbol{n}=\left(n^{1}, n^{2}\right) \in \mathbb{N}^{2}$ um estado qualquer. As taxas associadas ao processo $\left(N_{t}\right)_{t \geq 0}$ são dadas por

$$
\begin{array}{ll}
\lambda\left(\boldsymbol{n}, \boldsymbol{n}+\mathbf{1}_{j}\right)=(\mu-a) / 2, & \text { para } j=1,2 \text { e } \boldsymbol{n} \in \mathbb{N}^{2}, \\
\lambda\left(\boldsymbol{n}, \boldsymbol{n}-\mathbf{1}_{j}\right)=\mu, & \text { para } j=\min \{1,2\} \text { tal que } n^{j}>0 .
\end{array}
$$

Para um melhor entendimento, segue uma representação pictorial do processo.

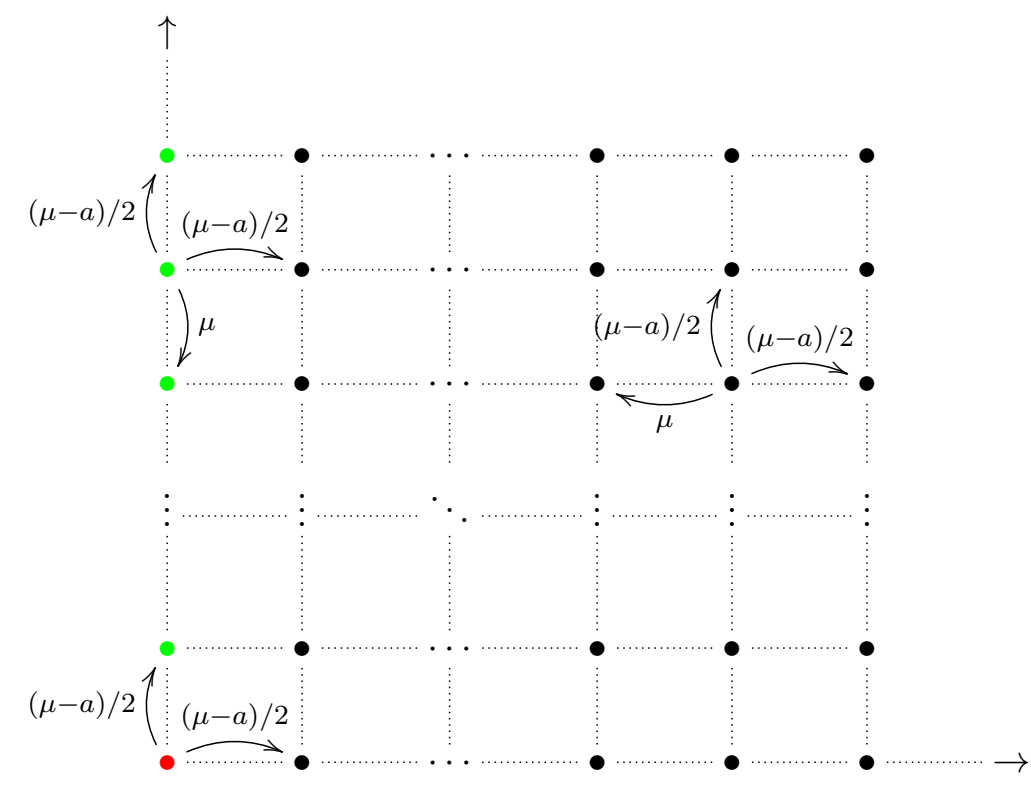

Figura 2.2: Representação pictorial dos estados e taxas para o processo $\left(N_{t}\right)_{t \geq 0}$. Nessa grade, cada ponto representa um estado e a variação das cores ocorre em função do comportamento das taxas de saída. O estado $(0,0)$ é representado pelo ponto vermelho.

Observação: Note que as taxas de saída de um determinado estado variam em função do próprio estado. Isso ocorre por duas razões: a reflexão do processo na origem, como já ocorria no caso anterior; e a correlação entre $N_{t}^{1}$ e $N_{t}^{2}$, já que uma espécie viva com aptidão no subintervalo mais alto só será extinta se, no instante da extinção, não existirem espécies com aptidões no subintervalo mais baixo.

Denotemos por $\pi_{2}$ a medida invariante associada ao processo $\left(N_{t}\right)_{t \geq 0}$ e seja $\pi_{2}(m, l):=\pi_{2}\left(n^{1}, n^{2}\right)=$ $\pi_{2}(\boldsymbol{n})$. Logo, temos as seguintes equações de balanceamento:

$$
\begin{gathered}
\pi_{2}(0,0)(\mu-a)=\pi_{2}(1,0) \mu+\pi_{2}(0,1) \mu ; \\
\pi_{2}(m, 0)(2 \mu-a)=\pi_{2}(m+1,0) \mu+\pi_{2}(m-1,0)(\mu-a) / 2, \text { para } m>0 ; \\
\pi_{2}(0, l)(2 \mu-a)=\pi_{2}(0, l+1) \mu+\pi_{2}(0, l-1)(\mu-a) / 2+\pi_{2}(1, l) \mu, \text { para } l>0 ;
\end{gathered}
$$

e, para $m>0$ e $l>0$,

$$
\pi_{2}(m, l)(2 \mu-a)=\pi_{2}(m+1, l) \mu+\pi_{2}(m-1, l)(\mu-a) / 2+\pi_{2}(m, l-1)(\mu-a) / 2 .
$$


Nosso principal objetivo nesta subseção é caracterizar $\pi_{2}$. No entanto, como veremos adiante, essa tarefa não será tão simples.

Da equação 2.20 e do fato de $\pi_{2}(0,0)=\pi_{1}(0)=a / \mu$, temos

$$
\pi_{2}(1,0)+\pi_{2}(0,1)=\frac{a}{\mu}\left(1-\frac{a}{\mu}\right)
$$

como deveria ser, pois sempre que o processo $\left(N_{t}\right)_{t \geq 0}$ visita os estados $(0,1)$ ou $(1,0)$, necessariamente o processo $\left(S_{t}\right)_{t \geq 0}$ visita o estado 1 .

Rearranjando o termos da equação 2.21 , temos

$$
\pi_{2}(m+1,0)-\left(2-\frac{a}{\mu}\right) \pi_{2}(m, 0)+\left(\frac{1}{2}-\frac{a}{2 \mu}\right) \pi_{2}(m-1,0)=0, \text { para } m>0 ;
$$

uma equação de diferenças finitas homogênea de segunda ordem.

Proposição 2.3.4. A medida invariante $\pi_{2}$ deve ser tal que para todo $m$ natural,

$$
\pi_{2}(m, 0)=\frac{a}{\mu}\left(\frac{q-\sqrt{q^{2}-4 r}}{2}\right)^{m},
$$

onde $q=2-a / \mu$ e $r=1 / 2-a / 2 \mu$.

Demonstração. Associada à equação 2.25, temos a equação característica

$$
y^{2}-q y+r=0,
$$

onde $q=2-a / \mu$ e $r=1 / 2-a / 2 \mu$, cujas raízes são dadas por

$$
\lambda_{1}=\frac{q+\sqrt{q^{2}-4 r}}{2} \quad \text { e } \quad \lambda_{2}=\frac{q-\sqrt{q^{2}-4 r}}{2} .
$$

Uma vez que $\Delta=q^{2}-4 r=1+(\mu-a)^{2} / \mu^{2}>0, \lambda_{1}$ e $\lambda_{2}$ são raízes reais e distintas. Logo, pela teoria de equações de diferenças finitas - vide (Elaydi, 2005), Capítulo 2, Seção 2.3 - segue que a solução geral para a equação 2.25 é dada por

$$
\pi_{2}=A \lambda_{1}^{m}+B \lambda_{2}^{m}
$$

onde $A$ e $B$ são constantes. Como $\lambda_{1}>1$, conforme $m \nearrow \infty$, teríamos $\lambda_{1}^{m} A \nearrow+\infty$ se $A>0$ e $\lambda_{1}^{m} A \rightarrow-\infty$ se $A<0$, um absurdo, já que $\pi_{2}$ é uma medida de de probabilidade. Daí, $A=0$ e a solução fica dada por

$$
\pi_{2}(m, 0)=B \lambda_{2}^{m} .
$$

Agora, basta conhecer o valor da constante $B$. Ao notar que $\pi_{2}(0,0)=\lambda_{2}^{0} B=B$, segue que $B=a / \mu$, o que finaliza a demonstração.

Vamos agora tentar obter a solução para $\pi_{2}(m, 1)$. Para tal, rearranjamos a equação 2.23 , de modo a ter

$$
\pi_{2}(m+1, l)-q \pi_{2}(m, l)+r \pi_{2}(m-1, l)+r \pi_{2}(m, l-1)=0 \text {, para } m>0 \text { e } l>0,
$$


$\operatorname{com} q=2-a / \mu$ e $r=1 / 2-a / 2 \mu$, assim como na Proposição 2.3.4. Fazendo $l=1$ em 2.31, segue que

$$
\pi_{2}(m+1,1)-q \pi_{2}(m, 1)+r \pi_{2}(m-1,1)+r \pi_{2}(m, 0)=0 ; \text { para } m>0,
$$

uma equação de diferenças finitas de segunda ordem não-homogênea.

Proposição 2.3.5. A medida invariante $\pi_{2}$ deve ser tal que para todo $m$ natural,

$$
\pi_{2}(m, 1)=\frac{a}{\mu}\left(1-\frac{a}{\mu}-\lambda\right) \lambda^{m}+\frac{a}{\mu} \frac{r}{r-\lambda^{2}} m \lambda^{m+1},
$$

$\operatorname{com} q=2-a / \mu, r=1 / 2-a / 2 \mu$ e $\lambda=\left(q-\sqrt{q^{2}-4 r}\right) / 2$.

Demonstração. Substituindo $\pi_{2}(m, 0)$ na equação 2.32 e fazendo $b_{m}=\pi_{2}(m, 1), \lambda=\left(\frac{q-\sqrt{q^{2}-4 r}}{2}\right)$, $c=a / \mu$, temos

$$
b_{m+1}-q b_{m}+r b_{m-1}+r c \lambda^{m}=0, \text { para } m>0,
$$

Da teoria de equações de diferenças finitas, segue que a candidata a solução particular seria

$$
b_{m}=d m \lambda^{m}
$$

onde $d$ é uma constante a ser conhecida. Substituindo a última na penúltima, segue que

$$
\begin{gathered}
d(m+1) \lambda^{m+1}-q d m \lambda^{m}+r d(m-1) \lambda^{m-1}+r c \lambda^{m}=0 \quad \Leftrightarrow \\
d m \lambda^{2}+d \lambda^{2}-q d m \lambda+r d m-r d+r c \lambda=0 \quad \Leftrightarrow \\
d m\left(\lambda^{2}-q \lambda+r\right)+d \lambda^{2}-r d+r c \lambda=0 \quad \Leftrightarrow \\
d\left(r-\lambda^{2}\right)=r c \lambda \quad \Leftrightarrow \\
d=\frac{r c \lambda}{r-\lambda^{2}} .
\end{gathered}
$$

Ademais, a solução para a equação homogênea seria dada por

$$
b_{m}^{h}=c \lambda^{m} .
$$

Aplicando os mesmos argumentos da Proposição 2.3.4, temos

$$
b_{m}^{h}=\pi_{2}(0,1) \lambda^{m} .
$$

Usando mais uma vez o resultado da Proposição 2.3.4 e a relação estabelecida pela equação 2.24, descobre-se que

$$
\pi_{2}(0,1)=\frac{a}{\mu}\left(1-\frac{a}{\mu}-\lambda\right) .
$$

Portanto, a solução geral é dada por

$$
\pi_{2}(m, 1)=\frac{a}{\mu}\left(1-\frac{a}{\mu}-\lambda\right) \lambda^{m}+\frac{a}{\mu} \frac{r}{r-\lambda^{2}} m \lambda^{m+1}
$$

A ideia para a caracterização de $\pi_{2}$ seria basicamente a seguinte: determinar $\pi_{2}(m, l)$ de modo iterativo, buscando algum padrão. Até aqui, já o fizemos para $l=0$ e $l=1$. O próximo passo seria para $l=2$. Apesar de possível, essa tarefa se mostra muito enfadonha sem perspectivas de uma 
função de resultado geral. O motivo para não continuarmos nossos cálculos é que tudo que haverá adiante dependerá basicamente do que já se conseguiu.

\subsection{Convergência em distribuição de $\mathcal{X}_{t}^{\prime}$}

Para estudar a convergência da distribuição de $\mathcal{X}_{t}^{\prime}$ quando $t \nearrow \infty$ como um processo pontual simples, vamos adotar o seguinte ponto de vista, com a seguinte descrição de $\mathcal{X}_{t}^{\prime}$ para cada $t$ fixo. Em primeiro lugar, vamos fixar $a \in(0, \mu)$ e considerar

$$
\mathcal{X}_{t}^{\prime \prime}=\mathcal{X}_{t}^{\prime} \cap[0, \mu-a] .
$$

Suporemos que $\mathcal{X}_{0}^{\prime \prime}=\emptyset$. Então

$$
\mathcal{X}_{t}^{\prime \prime}=\emptyset \quad \text { ou } \quad \mathcal{X}_{t}^{\prime \prime}=\left\{Y_{t}^{1}, \ldots, Y_{t}^{K_{t}}\right\}
$$

onde $Y_{t}^{1}, \ldots, Y_{t}^{K_{t}}$ são os $K_{t}$ pontos que constituem $\mathcal{X}_{t}^{\prime \prime}$.

Vamos representar $\mathcal{X}_{t}^{\prime \prime}$ como função de distribuição, definida a seguir. Fixado $t \geq 0$ e $x \in[0, \mu-a]$,

$$
F_{t}^{\prime \prime}=F_{t, a}^{\prime \prime}(x)= \begin{cases}\sum_{i=1}^{K_{t}} \mathbb{1}\left\{Y_{t}^{i} \leq x\right\}, & \text { se } \mathcal{X}_{t}^{\prime \prime} \neq \emptyset \\ 0, & \text { caso contrário. }\end{cases}
$$

Notemos que para cada $t \geq 0, F_{t}^{\prime \prime}:[0, \mu-a] \rightarrow \mathbb{N}$ é contínua à direita com limite a esquerda (cadlag). Temos então que para cada $t \geq 0, F_{t}^{\prime \prime}$ está no espaço de Skorohod $\mathscr{S}$ de funções cadlag definidas em $[0, \mu-a]$ à valores em $\mathbb{N}$ com a métrica $J_{1}$ - vide (Billingsley, 1968), Capítulo 3 e (Ethier e Kurtz, 1986), Capítulo 3.

Vamos a seguir mostrar que a distribuição de $F_{t}^{\prime \prime}$ converge em $\mathscr{S}$ à distribuição de um objeto $F_{\infty}^{\prime \prime}$, que será então necessariamente uma função de distribuição definida em $[0, \mu-a]$ à valores em $\mathbb{N}$ como veremos quase certamente finita. Temos então que o conjunto de pontos $\mathcal{X}_{\infty}^{\prime \prime}=\left\{Y_{\infty}^{1}, \ldots, Y_{\infty}^{K_{\infty}}\right\}$ tem que

$$
F_{\infty}^{\prime \prime}=F_{\infty, a}^{\prime \prime}(x)=\sum_{i=1}^{K_{\infty}} \mathbb{1}\left\{Y_{\infty}^{i} \leq x\right\}
$$

faz papel de limite em distribuição de $\mathcal{X}_{t}^{\prime \prime}$ quando $t \nearrow \infty$. Para estabelecer a convergência em distribuição de $\mathcal{X}_{t}^{\prime \prime}$ quando $t \nearrow \infty$, vamos verificar os seguintes critérios.

Teorema 2.4.1 (Critério para Existência). Existe um elemento aleatório em $\mathscr{S}$ com distribuições finito-dimensionais dada por $\nu_{x_{1} \ldots x_{k}}$, uma vez que as mesmas sejam consistentes; tal que

$$
\nu_{x_{1}, x, x_{2}}\left\{\left(m_{1}, m, m_{2}\right):\left|m-m_{1}\right| \geq \lambda,\left|m_{2}-m\right| \geq \lambda\right\} \leq \frac{1}{\lambda^{2 \gamma}}\left[G\left(x_{2}\right)-G\left(x_{1}\right)\right]^{2 \alpha}
$$

para $x_{1} \leq x \leq x_{2}$, onde $\gamma \geq 0, \alpha>1 / 2$, e $G$ é uma função não-decrescente contínua definida em $[0, \mu-a] ; \mathrm{e}$

$$
\lim _{\delta \downarrow 0} \nu_{x, x+\delta}\left\{\left(m_{1}, m_{2}\right):\left|m_{1}-m_{2}\right| \geq \epsilon\right\}=0
$$

para $0 \leq x<\mu-a$.

Demonstração. A demostração deste Teorema pode ser vista em (Billingsley, 1968), Capítulo 3, Teorema 15.7. 
Teorema 2.4.2 (Critério para Convergência). Suponha que

$$
\left(F_{n}\left(x_{1}\right), \ldots, F_{n}\left(x_{k}\right)\right) \stackrel{\mathscr{D}}{\rightarrow}\left(F_{\infty}\left(x_{1}\right), \ldots, F_{\infty}\left(x_{k}\right)\right)
$$

vale sempre que $x_{i} \in T_{F_{\infty}}, i=1, \ldots, k ;$ que $\mathbb{P}\left\{F(\mu-a) \neq F\left((\mu-a)^{-}\right)\right\}=0$, e que

$$
\mathbb{P}\left\{\left|F_{n}(x)-F_{n}\left(x_{1}\right)\right| \geq \lambda,\left|F_{n}\left(x_{2}\right)-F_{n}(x)\right| \geq \lambda\right\} \leq \frac{1}{\lambda^{2 \gamma}}\left[G\left(x_{2}\right)-G\left(x_{1}\right)\right]^{2 \alpha}
$$

para $x_{1} \leq x \leq x_{2}$ e $n \geq 1$, onde $\gamma \geq 0, \alpha>1 / 2, \lambda$ positivo, e $G$ uma função não-decrescente contínua definida em $[0, \mu-a]$. Então $F_{n} \stackrel{\mathscr{D}}{\rightarrow} F_{\infty}$.

Demonstração. A demostração deste Teorema pode ser vista em (Billingsley, 1968), Capítulo 3, Teorema 15.6..

Vamos agora mostrar que existe um objeto $F_{\infty}^{\prime \prime}$ tal que a distribuição de $F_{t}^{\prime \prime}$ converge em $\mathscr{S}$ à distribuição de $F_{\infty}^{\prime \prime}$.

Teorema 2.4.3. Existe um objeto $F_{\infty}^{\prime \prime}$ em $\mathscr{S}$ tal que $F_{t}^{\prime \prime} \stackrel{\mathscr{D}}{\rightarrow} F_{\infty}^{\prime \prime}$.

Demonstração. Para $m_{i} \in \mathbb{N}$ e $x_{i} \in(0, \mu-a), i \in\{1, \ldots, k\}$, com $m_{i} \leq m_{i+1}$ e $x_{i}<x_{i+1}$, considere o evento $\pi_{x_{1} \ldots x_{k}, t}^{m_{1} \ldots m_{k}}=\left\{F_{t}^{\prime \prime}\left(x_{1}\right)=m_{1}, F_{t}^{\prime \prime}\left(x_{2}\right)=m_{2}, \ldots, F_{t}^{\prime \prime}\left(x_{k}\right)=m_{k}\right\}$. Note que

$$
\begin{aligned}
\mathbb{P}\left\{\pi_{x_{1} \ldots x_{k}, t}^{m_{1} \ldots m_{k}}\right\} & \underset{1}{\longrightarrow} \underset{t \nearrow \infty}{\longrightarrow} \pi_{x_{1} \ldots x_{k}}\left(m_{1}, m_{2}-m_{1}, \ldots, m_{k}-m_{k-1}\right) \\
=: & \nu_{x_{1} \ldots x_{k}}\left(m_{1}, m_{2}, \ldots, m_{k}\right)
\end{aligned}
$$

onde $I_{k}=\left[x_{k-1}, x_{k}\right)$ com $x_{0}=0, \pi_{x_{1} \ldots x_{k}}$ é dada e consiste da medida invariante associada ao processo $\left(W_{t}\right)_{t \geq 0} \operatorname{com} W_{t}=\left(\left|\mathcal{X}_{t}^{\prime \prime} \cap I_{1}\right|,\left|\mathcal{X}_{t}^{\prime \prime} \cap I_{2}\right|, \ldots,\left|\mathcal{X}_{t}^{\prime \prime} \cap I_{k}\right|\right)^{1}$. Definida desta forma, $\nu_{x_{1} \ldots x_{k}}$ é consistente. Para ver isso, seja $M>0$ real e note que

$$
\begin{aligned}
& \nu_{x_{1} \ldots x_{k-1}}\left(m_{1}, \ldots, m_{k-1}\right)=\lim _{t \nearrow \infty} \mathbb{P}\left\{\left|\mathcal{X}_{t}^{\prime \prime} \cap I_{1}\right|=m_{1}, \ldots,\left|\mathcal{X}_{t}^{\prime \prime} \cap I_{k-1}\right|=m_{k-1}-m_{k-2}\right\} \\
& =\lim _{t \nearrow \infty} \sum_{m_{k} \leq\lfloor M\rfloor} \mathbb{P}\left\{\left|\mathcal{X}_{t}^{\prime \prime} \cap I_{1}\right|=m_{1}, \ldots,\left|\mathcal{X}_{t}^{\prime \prime} \cap I_{k-1}\right|=m_{k-1}-m_{k-2},\left|\mathcal{X}_{t}^{\prime \prime} \cap I_{k}\right|=m_{k}-m_{k-1}\right\} \\
& \quad+\lim _{t \nearrow \infty} \sum_{m_{k}>\lfloor M\rfloor} \mathbb{P}\left\{\left|\mathcal{X}_{t}^{\prime \prime} \cap I_{1}\right|=m_{1}, \ldots,\left|\mathcal{X}_{t}^{\prime \prime} \cap I_{k-1}\right|=m_{k-1}-m_{k-2},\left|\mathcal{X}_{t}^{\prime \prime} \cap I_{k}\right|=m_{k}-m_{k-1}\right\} \\
& =\sum_{m_{k} \leq\lfloor M\rfloor} \lim _{t \nearrow \infty} \mathbb{P}\left\{\left|\mathcal{X}_{t}^{\prime \prime} \cap I_{1}\right|=m_{1}, \ldots,\left|\mathcal{X}_{t}^{\prime \prime} \cap I_{k-1}\right|=m_{k-1}-m_{k-2},\left|\mathcal{X}_{t}^{\prime \prime} \cap I_{k}\right|=m_{k}-m_{k-1}\right\} \\
& \quad+\lim _{t \nearrow \infty} \sum_{m_{k}>\lfloor M\rfloor} \mathbb{P}\left\{\left|\mathcal{X}_{t}^{\prime \prime} \cap I_{1}\right|=m_{1}, \ldots,\left|\mathcal{X}_{t}^{\prime \prime} \cap I_{k-1}\right|=m_{k-1}-m_{k-2},\left|\mathcal{X}_{t}^{\prime \prime} \cap I_{k}\right|=m_{k}-m_{k-1}\right\} \\
& =\sum_{m_{k} \leq\lfloor M\rfloor} \pi_{x_{1} \ldots x_{k-1} x_{k}}\left(m_{1}, \ldots, m_{k-1}-m_{k-2}, m_{k}-m_{k-1}\right) \\
& \quad+\lim _{t \nearrow \infty} \sum_{m_{k}>\lfloor M\rfloor} \mathbb{P}\left\{\left|\mathcal{X}_{t}^{\prime \prime} \cap I_{1}\right|=m_{1}, \ldots,\left|\mathcal{X}_{t}^{\prime \prime} \cap I_{k-1}\right|=m_{k-1}-m_{k-2},\left|\mathcal{X}_{t}^{\prime \prime} \cap I_{k}\right|=m_{k}-m_{k-1}\right\} .
\end{aligned}
$$

\footnotetext{
${ }^{1}$ Para verificar que $\pi_{x_{1} \ldots x_{k}}$ existe e é única, basta mostrar que $\left(W_{t}\right)_{t \geq 0}$ é recorrente positivo. Essa tarefa pode ser facilmente executada por meio de uma simples atualização dos argumentos das Proposições 2.3.1 e 2.3.2.
} 
Uma vez que $M$ é arbitrário, podemos tomar o limite quando $M \nearrow \infty$ em ambos os lados da equação acima, o que resulta em

$$
\begin{aligned}
\nu_{x_{1} \ldots x_{k-1}}\left(m_{1}, \ldots, m_{k-1}\right) & =\sum_{m_{k} \in \mathbb{N}} \pi_{x_{1} \ldots x_{k-1} x_{k}}\left(m_{1}, \ldots, m_{k-1}-m_{k-2}, m_{k}-m_{k-1}\right) \\
& =\sum_{m_{k} \in \mathbb{N}} \nu_{x_{1} \ldots x_{k-1} x_{k}}\left(m_{1}, \ldots, m_{k-1}, m_{k}\right),
\end{aligned}
$$

pois $\sum_{m_{k}>\lfloor M]} \mathbb{P}\left\{\left|\mathcal{X}_{t}^{\prime \prime} \cap I_{1}\right|=m_{1}, \ldots,\left|\mathcal{X}_{t}^{\prime \prime} \cap I_{k-1}\right|=m_{k-1}-m_{k-2},\left|\mathcal{X}_{t}^{\prime \prime} \cap I_{k}\right|=m_{k}-m_{k-1}\right\}$ é menor ou igual à $\sum_{m_{k}>\lfloor M\rfloor} \pi_{x_{k}}\left(m_{k}\right)=\left(x_{k} / \mu\right)^{\lfloor M\rfloor}$, que tende a zero quando $M \nearrow \infty$. Agora, para $\lambda$ positivo, seja $A_{t}\left(\lambda ; x_{1}, x, x_{2}\right):=\mathbb{P}\left\{\left|F_{t}^{\prime \prime}(x)-F_{t}^{\prime \prime}\left(x_{1}\right)\right| \geq \lambda,\left|F_{t}^{\prime \prime}\left(x_{2}\right)-F_{t}^{\prime \prime}(x)\right| \geq \lambda\right\}$. Note que

$$
\begin{aligned}
A_{t}\left(\lambda ; x_{1}, x, x_{2}\right) & \leq \mathbb{P}\left\{\left|F_{t}^{\prime \prime}(x)-F_{t}^{\prime \prime}\left(x_{1}\right)\right| \geq 1,\left|F_{t}^{\prime \prime}\left(x_{2}\right)-F_{t}^{\prime \prime}(x)\right| \geq 1\right\} \\
& \leq \mathbb{P}\left\{\left|F_{t}^{\prime \prime}\left(x_{2}\right)-F_{t}^{\prime \prime}\left(x_{1}\right)\right| \geq 2\right\} \\
& \leq \sum_{m \geq 0} \sum_{l \geq 2} \pi_{x_{1} x_{2}}(m, l) \\
& =1-\sum_{m \in \mathbb{N}} \pi_{x_{1} x_{2}}(m, 0)-\sum_{m \in \mathbb{N}} \pi_{x_{1} x_{2}}(m, 1)=: A_{x_{1}, x_{2}},
\end{aligned}
$$

onde a terceira desigualdade se justifica pelo fato de que, por construção, o evento $\left\{\mid F_{t}^{\prime \prime}\left(x_{2}\right)-\right.$ $\left.F_{t}^{\prime \prime}\left(x_{1}\right) \mid \geq 2\right\}$ está restrito à condição inicial vazia, o que o torna menos (no máximo, tão) provável quanto à quando restrito a condição inicial não-vazia determinada pela medida invariante $\pi_{x_{1} x_{2}}$. A fim de determinar $A_{x_{1}, x_{2}}$, basta caracterizar, para todo $m \in \mathbb{N}, \pi_{x_{1} x_{2}}(m, 0)$ e $\pi_{x_{1} x_{2}}(m, 1)$. Vamos à isso. Pode-se verificar sem maiores dificuldades que as taxas associadas ao processo $W_{t}=\left(\mid \mathcal{X}_{t}^{\prime \prime} \cap\right.$ $\left.\left[0, x_{1}\right)|,| \mathcal{X}_{t}^{\prime \prime} \cap\left[x_{1}, x_{2}\right) \mid\right)$ são dadas por

$$
\begin{array}{ll}
\lambda\left(n, n+\mathbf{1}_{j}\right)=\left(x_{j}-x_{j-1}\right), & \text { para } j=1,2, x_{0}=0 \text { e } n=\left(n^{1}, n^{2}\right) \in \mathbb{N}^{2}, \\
\lambda\left(n, n-\mathbf{1}_{j}\right)=\mu, & \text { para } j=\min \{1,2\} \text { tal que } n^{j}>0 .
\end{array}
$$

Por uma atualização dos argumentos na Subseção 2.3.1 para o caso $k=2$, obtém-se que,

$$
\begin{gathered}
\pi_{x_{1} x_{2}}(m, 0)=\left(1-\frac{x_{1}+\delta}{\mu}\right) \eta^{m}, \\
\pi_{x_{1} x_{2}}(m, 1)=\left(1-\frac{x_{1}+\delta}{\mu}\right)\left(\frac{x_{1}+\delta}{\mu}-\eta\right) \eta^{m}+\left(1-\frac{x_{1}+\delta}{\mu}\right) \frac{\delta}{x_{1}-\mu \eta^{2}} m \eta^{m+1},
\end{gathered}
$$

para $m \in \mathbb{N}$, onde $\delta=x_{2}-x_{1}$, e $\eta=\frac{\mu+x_{1}+\delta-\sqrt{\left(\mu+x_{1}+\delta\right)^{2}-4 x_{1} \mu}}{2 \mu}$. Daí, segue que

$$
\begin{aligned}
\sum_{m \in \mathbb{N}} \pi_{x_{1} x_{2}}(m, 0) & =\sum_{m=0}^{\infty}\left(1-\frac{x_{1}+\delta}{\mu}\right) \eta^{m}=\frac{1}{1-\eta}\left(1-\frac{x_{1}+\delta}{\mu}\right) \\
& =2 \times \frac{\mu-x_{1}-\delta}{\mu-x_{1}-\delta+\sqrt{\left(\mu-x_{1}\right)^{2}+\delta\left(\delta+2 x_{1}+2 \mu\right)}} \\
& =: \quad f\left(x_{1}, \delta\right),
\end{aligned}
$$




$$
\begin{aligned}
\sum_{m \in \mathbb{N}} \pi_{x_{1} x_{2}}(m, 1) & =\sum_{m=0}^{\infty}\left(1-\frac{x_{1}+\delta}{\mu}\right)\left(\frac{x_{1}+\delta}{\mu}-\eta\right) \eta^{m} \\
& +\sum_{m=0}^{\infty}\left(1-\frac{x_{1}+\delta}{\mu}\right) \frac{\delta}{x_{1}-\mu \eta^{2}} m \eta^{m+1} \\
& =\left(1-\frac{x_{1}+\delta}{\mu}\right)\left(\frac{x_{1}+\delta}{\mu}-\eta\right) \frac{1}{1-\eta} \\
& +\left(1-\frac{x_{1}+\delta}{\mu}\right) \frac{\delta}{x_{1}-\mu \eta^{2}} \frac{\eta^{2}}{(1-\eta)^{2}}=: g\left(x_{1}, \delta\right)+h\left(x_{1}, \delta\right)
\end{aligned}
$$

Fazendo a substituição pelo real valor de $\eta$ temos, após um certo trabalho algébrico, que

$$
\begin{gathered}
g\left(x_{1}, \delta\right)=\frac{4 \delta\left(\mu-x_{1}-\delta\right)}{\left(\sqrt{\left(\mu+x_{1}+\delta\right)^{2}-4 x_{1} \mu}+\mu-x_{1}-\delta\right)^{2}}, \text { e que, } \\
h\left(x_{1}, \delta\right)=\frac{4 \delta\left(\mu-x_{1}-\delta\right)\left(\mu+x_{1}+\delta-\sqrt{\left(\mu+x_{1}+\delta\right)^{2}-4 x_{1} \mu}\right)^{2}}{\left(\sqrt{\left(\mu+x_{1}+\delta\right)^{2}-4 x_{1} \mu}+\mu-x_{1}-\delta\right)^{2}\left(4 x_{1} \mu-\left(\mu+x_{1}+\delta-\sqrt{\left(\mu+x_{1}+\delta\right)^{2}-4 x_{1} \mu}\right)^{2}\right)}
\end{gathered}
$$

Logo,

$$
A_{x_{1}, x_{2}}=1-f\left(x_{1}, \delta\right)-g\left(x_{1}, \delta\right)-h\left(x_{1}, \delta\right)
$$

Fixado $x_{1}$, o lado esquerdo da inequação acima é uma função analítica. Assim, podemos expandila e representá-la por meio de sua respectiva série de MacLaurin. Após algumas manipulações algébricas, obtém-se que

$$
\begin{aligned}
& f\left(x_{1}, \delta\right)=1-\frac{\mu}{\left(\mu-x_{1}\right)^{2}} \delta+c_{1} \delta^{2}+o\left(\delta^{2}\right), \\
& g\left(x_{1}, \delta\right)=\frac{\mu-x_{1}}{\left(\mu-x_{1}\right)^{2}} \delta+c_{2} \delta^{2}+o\left(\delta^{2}\right), \\
& h\left(x_{1}, \delta\right)=\frac{x_{1}}{\left(\mu-x_{1}\right)^{2}} \delta+c_{3} \delta^{2}+o\left(\delta^{2}\right),
\end{aligned}
$$

onde $c_{1}, c_{2}$ e $c_{3}$ são constantes. Isto é, existe uma constante $c$ tal que

$$
A_{t}\left(\lambda ; x_{1}, x, x_{2}\right) \leq A_{x_{1}, x_{2}}=c \delta^{2}+o\left(\delta^{2}\right) .
$$

Da relação acima e do fato de que $o\left(\delta^{2}\right)<r \delta^{2}$ para alguma constante $0<r<\infty$, para $s=$ $\max \{\sqrt{c}, r, 1\}, G(x)=s x, \lambda$ positivo, $\gamma=0$ e $\alpha=1$, segue que

$$
A_{t}\left(\lambda ; x_{1}, x, x_{2}\right) \leq A_{x_{1}, x_{2}} \leq \frac{1}{\lambda^{2 \gamma}}\left[G\left(x_{2}\right)-G\left(x_{1}\right)\right]^{2 \alpha} .
$$

Ademais, uma vez que $\forall \epsilon>0$,

$$
\begin{aligned}
\nu_{x_{1}, x_{1}+\delta}\left\{\left(m_{1}, m_{2}\right):\left|m_{1}-m_{2}\right| \geq \epsilon\right\} & \leq \nu_{x_{1}, x_{1}+\delta}\left\{\left(m_{1}, m_{2}\right):\left|m_{1}-m_{2}\right| \geq 1\right\} \\
& \leq \sum_{m \geq 0} \sum_{l \geq 1} \nu_{x_{1} x_{1}+\delta}(m, m+l) \\
& =1-\sum_{m \in \mathbb{N}} \pi_{x_{1} x_{1}+\delta}(m, 0)=1-f\left(x_{1}, \delta\right),
\end{aligned}
$$

segue, a partir de 2.57 , que

$$
\lim _{\delta \downarrow 0} \nu_{x_{1}, x_{1}+\delta}\left\{\left(m_{1}, m_{2}\right):\left|m_{1}-m_{2}\right| \geq \epsilon\right\}=0 .
$$


Para concluir usamos a consistência de $\nu_{x_{1} \ldots x_{k}}$ e 2.63 para estabelecer, usando do Teorema 2.4.1, a existência de $F_{\infty}^{\prime \prime}$ em $\mathscr{S}$ tal que

$$
\nu_{x_{1} \ldots x_{k}}\left(m_{1}, m_{2}, \ldots, m_{k}\right)=\mathbb{P}\left\{F_{\infty}^{\prime \prime}\left(x_{1}\right)=m_{1}, F_{\infty}^{\prime \prime}\left(x_{2}\right)=m_{2}, \ldots, F_{\infty}^{\prime \prime}\left(x_{k}\right)=m_{k}\right\} .
$$

Em seguida, de 2.48 e 2.61 , obtemos do Teorema 2.4 .2 que $F_{t}^{\prime \prime} \stackrel{\mathscr{D}}{\rightarrow} F_{\infty}^{\prime \prime}$.

Antes de prosseguir, ressaltamos que a validade de 2.63 implica que $F_{\infty}^{\prime \prime}$ é um processo pontual simples para todo $a \in(0, \mu)$, dado que a probabilidade de existir mais de um ponto em qualquer subintervalo de comprimento $\delta$ contido em $(0, \mu-a)$ vai à zero quando $\delta \searrow 0$. Ademais, mencionamos que há na literatura uma outra forma de abordar o problema, onde $\left(\mathcal{X}_{t}^{\prime \prime}\right)_{t \geq 0}$ pode ser visto como um processo de nascimento e morte espacial - vide (Preston, 1975). Não utilizamos essa última abordagem pois o conhecimento sobre a mesma ocorreu quase ao fim do término deste trabalho.

Agora, da mesma forma que para $\mathcal{X}_{t}^{\prime \prime}$ podemos descrever $\mathcal{X}_{t}^{\prime}$ a partir de sua função distribuição $F_{t}^{\prime}$. Dados $a, a^{\prime} \in(0, \mu)$ com $a^{\prime}<a$, é fácil ver que

$$
\left.F_{t}^{\prime}\right|_{[0, \mu-a]}=\left.F_{t, a^{\prime}}^{\prime \prime}\right|_{[0, \mu-a]}=F_{t, a}^{\prime \prime}
$$

Do que segue que para cada $0<b<\mu$,

$$
\left.F_{t}^{\prime}\right|_{[0, b]} \stackrel{\mathscr{D}}{\rightarrow} F_{\infty, b}^{\prime},
$$

satisfazendo $\left.F_{\infty, b^{\prime}}^{\prime}\right|_{[0, b]}=F_{\infty, b}^{\prime}$ para $0<b<b^{\prime}<\mu$.

Da consistência acima, obtemos a existência de $F_{\infty}^{\prime}:[0, \mu) \rightarrow \mathbb{N}$ tal que

$$
F_{\infty}^{\prime}(x)=F_{\infty, x}^{\prime}(x),
$$

que está bem definida em $[0, \mu)$. Vamos tomá-lo como o limite em distribuição de $F_{t}^{\prime}$ e logo de $\mathcal{X}_{t}^{\prime}$.

\subsection{Propriedades da medida limite}

Nessa seção, apresentamos algumas propriedades de $\mathcal{X}_{\infty}^{\prime}$ derivadas da representação de $\mathcal{X}_{\infty}^{\prime}$ por $F_{\infty}^{\prime}$

Proposição 2.5.1. Decorre que:

1) $\left|\mathcal{X}_{\infty}^{\prime}\right|:=\lim _{b \nearrow \mu} F_{\infty, b}^{\prime}=\infty$ q.c.

2) Para $0 \leq a \leq b<\mu$,

a) $\mathbb{E}_{0}\left|\mathcal{X}_{\infty}^{\prime} \cap[a, b]\right|=\frac{\mu(b-a)}{(\mu-a)(\mu-b)}$,

b) $\mathbb{P}_{0}\left(\mathcal{X}_{\infty}^{\prime} \cap[a, b]=\emptyset\right)=\frac{2(\mu-b)}{\mu-b+\sqrt{(\mu-a)^{2}+(b-a)(b+a+2 \mu)}}$

Demonstração. Para mostrar 1), note que para $m \in \mathbb{N}$,

$$
\mathbb{P}\left(F_{\infty, b}^{\prime}=m\right)=\nu_{b}(m)=\pi_{b}(m)=(1-b / \mu)(b / \mu)^{m},
$$

ou seja, $F_{\infty, b}^{\prime}$ tem distribuição geométrica com parâmetro $1-b / \mu$. Daí, dado $b$, para $M>0$ real, segue que

$$
\mathbb{P}\left(F_{\infty, b}^{\prime}>M\right)=\sum_{m=\lceil M\rceil}^{\infty}\left(1-\frac{b}{\mu}\right)\left(\frac{b}{\mu}\right)^{m}=\left(\frac{b}{\mu}\right)^{\lceil M\rceil}
$$


Da relação acima, observe que para todo $b<\mu, F_{\infty, b}^{\prime}$ é finito quase certamente. Por outro lado, para $\left|\mathcal{X}_{\infty}^{\prime}\right|:=\lim _{b \nearrow_{\mu}} F_{\infty, b}^{\prime}$, segue que

$$
\mathbb{P}\left(\left|\mathcal{X}_{\infty}^{\prime}\right|>M\right)=1, \forall M
$$

isto é, $\left|\mathcal{X}_{\infty}^{\prime}\right| \stackrel{\text { q.c. }}{=} \infty$. Com isso, temos demostrada a propriedade 1). Note, portanto, que o valor $\mu$ é um ponto de acumulação.

Para mostrar 2.a), basta perceber que

$$
\begin{aligned}
\mathbb{E}_{0}\left|\mathcal{X}_{\infty}^{\prime} \cap[a, b]\right| & =\mathbb{E}_{0}\left(F_{\infty, b}^{\prime}-F_{\infty, a}^{\prime}\right) \\
& =\mathbb{E}_{0}\left(F_{\infty, b}^{\prime}\right)-\mathbb{E}_{0}\left(F_{\infty, a}^{\prime}\right) \\
& =\frac{b}{\mu-b}-\frac{a}{\mu-a}=\frac{\mu(b-a)}{(\mu-a)(\mu-b)} .
\end{aligned}
$$

Por fim, 2.b) segue do seguinte argumento:

$$
\begin{aligned}
\mathbb{P}_{0}\left(\mathcal{X}_{\infty}^{\prime} \cap[a, b]=\emptyset\right) & =\sum_{m=0}^{\infty} \mathbb{P}_{0}\left(F_{\infty, a}^{\prime}=m, F_{\infty, b}^{\prime}=m\right) \\
& =\sum_{m=0}^{\infty} \nu_{a, b}(m, m)=\sum_{m=0}^{\infty} \pi_{a, b}(m, 0) \\
& =\frac{2(\mu-b)}{\mu-b+\sqrt{(\mu-a)^{2}+(b-a)(b+a+2 \mu)}} .
\end{aligned}
$$

Para findar, argumentamos que para $\left|\mathcal{X}_{0}^{\prime}\right|<\infty,\left(\left|\mathcal{X}_{t}^{\prime \prime}\right|\right)_{t>0}$ retornará à origem após um tempo aleatório finito, uma vez que consiste de um processo de nascimento e morte recorrente positivo. Daí, conclui-se sem maiores dificuldades que, a partir de uma condição inicial localmente finita, permanecem válidos os resultados acima. 


\section{Capítulo 3}

\section{Sobre a espécie eterna}

Por resultados já conhecidos, sabe-se que todas as espécies que surgem com aptidões maiores que $\mu$ sobrevivem para sempre com probabilidade positiva - vide Guiol et al. (2013), Corolário 2.5 e/ou Observação 2.6. Nesse capítulo, iremos mostrar que espécies eternas surgem eventualmente com probabilidade 1. Após a verificação do eventual surgimento das mesmas, identificaremos seus respectivos instantes de nascimento por um processo pontual $\hat{\mathcal{P}}$ em $[0, \infty) \times[0,1]$ a ser definido. Explicitaremos a função zero de $\hat{\mathcal{P}}$ por meio de uma equação integral e apresentaremos estimativas para a taxa de decaimento (exponencial) da cauda da distribuição do tempo de surgimento da primeira espécie imortal a partir da condição inicial vazia.

\subsection{Infinitas espécies eternas}

Para cada $a \in[0,1]$ fixado, definamos

$$
M_{a, t}=\left|\mathcal{X}_{t} \cap[0, a)\right|,
$$

que indica o número de espécies vivas no instante $t$ com aptidões inferiores à $a$. Note que $\left\{M_{a, t}: t \geq 0\right\}$ é um processo de nascimento e morte em tempo contínuo com taxas de nascimento e morte $a$ e $\mu$, respectivamente.

Seja $\left\{M_{a, t}^{x}: t \geq 0\right\}$ um processo construído assim como o processo $\left\{M_{a, t}: t \geq 0\right\}$ tendo $x$ como valor inicial, isto é, $M_{a, 0}^{x}=x$. Ademais, seja $\sigma_{x}=\inf \left\{t>0: M_{a, t}^{x} \neq x\right\}$ o tempo até o primeiro salto do processo $\left\{M_{a, t}^{x}: t \geq 0\right\}$ e $T^{a, x, y}=\inf \left\{t>\sigma_{x}: M_{a, t}^{x}=y\right\}$ o tempo até sua primeira passagem ${ }^{1}$ pelo estado $y$.

Dos resultados de Processos Estocásticos - vide (Grimmett e Stirzaker, 2001) - sabe-se que:

1. Se $a \leq \mu$, então $\left\{M_{a, t}: t \geq 0\right\}$ é recorrente. Disto decorre que se $\mathcal{X}_{0}=\emptyset$, então quase certamente toda espécie com aptidão em $[0, \mu]$ morre eventualmente.

2. Para $a>\mu,\left\{M_{a, t}: t \geq 0\right\}$ é transitório, isto é, $\mathbb{P}\left(T^{a, x, x}=\infty\right)>0$, para todo $x \in \mathbb{N}$.

Ademais, vamos denotar por $\left\{L_{a, n}: n \geq 0\right\}$ a cadeia imersa de $\left\{M_{a, t}^{0}: t \geq 0\right\}$. Note que $\left\{L_{a, n}: n \geq 0\right\}$ é um passeio aleatório simples nos inteiros positivos com reflexão na origem, cujas probabilidades de salto à direita e à esquerda são iguais à $\frac{a}{a+\mu} \mathrm{e} \frac{\mu}{a+\mu}$, respectivamente.

Com isso, temos condições de provar a principal proposição desta seção, enunciada a seguir.

\footnotetext{
${ }^{1}$ Caso $x=y, T^{a, x, y}=T^{a, x, x}$ é dito o tempo de retorno do processo $\left\{M_{a, t}^{x}: t \geq 0\right\}$ ao estado $x$.
} 
Teorema 3.1.1. Existem infinitas espécies que sobrevivem para sempre quase certamente.

Demonstração. Para cada $a \in(\mu, 1]$, seja $N_{a}$ o número de retornos de $\left\{M_{a, t}^{0}: t \geq 0\right\}$ à origem. Então, $N_{a} \sim \operatorname{geo}(\rho)$, isto é, $N_{a}$ é uma variável aleatória com distribuição geométrica com parâmetro $\rho$, onde $\rho:=\mathbb{P}\left(T^{a, 0,0}=\infty\right)>0$. A partir de $\mathbb{P}\left(N_{a}<k\right)=1-(1-\rho)^{k}, k \in \mathbb{N}$, segue que $\mathbb{P}\left(N_{a}<\infty\right)=1$, isto é, o número de vezes que $\left\{M_{a, t}^{0}: t \geq 0\right\}$ retorna à origem é finito quase certamente. O tempo aleatório até a ocorrência do último retorno é dado por

$$
\tau_{a}=\sum_{k=1}^{N_{a}} T_{a, k},
$$

onde, pela propriedade de Markov, $T_{a, 1}, \ldots, T_{a, N_{a}}$ são i.i.d. e independentes de $N_{a}$, e tal que

$$
T_{a, 1} \stackrel{\mathscr{P}}{=} \sum_{k=1}^{L_{a}} \xi_{a, k},
$$

onde $L_{a}$ é o tempo de retorno, dado que é finito, de $\left\{L_{a, n}: n \geq 0\right\}$ à origem e $\xi_{a, 1}, \ldots, \xi_{a, L_{a}}$ são variáveis aleatórias i.i.d. e independentes de $L_{a}, \operatorname{com} \xi_{a, 1} \sim \exp (a+\mu)$. Note que $\tau_{a}$ é uma variável aleatória finita quase certamente, uma vez que corresponde a uma soma quase certamente finita de variáveis aleatórias quase certamente finitas. A partir de $\tau_{a}$, basta esperar o surgimento da primeira espécie com aptidão abaixo de $a$, o que ocorre num tempo exponencialmente distribuído com taxa $a$ - digamos $\phi_{a_{\downarrow}}$ - que é finito quase certamente. Daí, $\left\{M_{a, t}^{0}: t \geq 0\right\}$ nunca mais retornará ao estado zero, isto é, as espécies com aptidões em $(a, 1]$ serão protegidas pelas com aptidões em $[0, a]$. Caso não existam espécies com aptidões acima de $a$, basta esperar pelo nascimento da próxima com essa característica, que surgirá de acordo com uma variável aleatória exponencial com taxa $(1-a)$ digamos $\phi_{a_{\uparrow}}$ - que é finita quase certamente. Logo, no instante

$$
\mathcal{S}_{a}=\tau_{a}+\phi_{a_{\downarrow}}+\phi_{a_{\uparrow}},
$$

teremos uma espécie que sobreviverá para sempre. Vale notar que $\mathcal{S}_{a}$ é uma cota superior para o tempo de surgimento da primeira espécie eterna. Ademais, todas as espécies que aparecerem depois desse tempo aleatório com aptidões acima de $a$ sobreviverão para sempre. Isto é suficiente para garantir que, quase certamente, surgirão infinitas espécies que sobrevivem para sempre.

Ademais, seja $\mathcal{S}_{a}^{-}=\tau_{a}+\phi_{a_{\downarrow}}$. Para todo $t>\mathcal{S}_{a}^{-}$, o conjunto de espécies eternas com aptidões acima de $a$ pode ser descrito como o conjunto de espécies vivas e com aptidões acima de $a$ em $\mathcal{S}_{a}^{-}$unido àquelas surgidas em $\left[\mathcal{S}_{a}^{-}, t\right] \times[a, 1]$, isto é, são dadas pelo conjunto

$$
\mathcal{X}_{a}^{\prime} \cup \tilde{\mathcal{X}}_{a, t},
$$

onde $\mathcal{X}_{a}^{\prime}=\left.\mathcal{X}_{\mathcal{S}_{a}^{-}}\right|_{(a, 1]}$ e $\tilde{\mathcal{X}}_{a, t}=\left.\mathcal{N}\right|_{\left(\mathcal{S}_{a}^{-}, t\right) \times(a, 1]}$. Note que dado $\mathcal{S}_{a}^{-}, \tilde{\mathcal{X}}_{a, t}$ é independente de $\mathcal{X}_{a}^{\prime}$ em $\left(\mathcal{S}_{a}^{-}, t\right) \times(a, 1]$ e é um processo de Poisson com taxa $1-a$. Com isto, temos o seguinte resultado.

Proposição 3.1.1. Partindo de uma condição inicial localmente finita, temos que

$$
\frac{1}{t}\left|\hat{\mathcal{X}}_{t}\right|_{I}|\rightarrow(1-a)| I \mid, \quad \text { quase certamente quando } t \nearrow \infty
$$

onde $\hat{\mathcal{X}}_{t}$ é o conjunto de espécies eternas no tempo $t$ e $I \subset(a, 1]$ é um intervalo fechado.

Demonstração. Para cada $a \in(\mu, 1]$ e $B \subset[0,1]$ localmente finito, fixemos $\mathcal{X}_{0}=B$ e definamos $\mathcal{R}_{a}^{-}$ como o tempo em que $\left\{M_{a, t}: t \geq 0\right\}$ salta do estado zero pela última vez, com a convenção de que valerá zero caso o salto não ocorra. Por um simples acoplamento de $\left\{M_{a, t}: t \geq 0\right\}$ e $\left\{M_{a, t}^{0}: t \geq 0\right\}$, 
é fácil ver que $\mathcal{R}_{a}^{-} \preceq \mathcal{S}_{a}^{-}$, isto é, $\mathcal{R}_{a}^{-}$é finito quase certamente. A partir daqui, basta notar que para todo $t>\mathcal{R}_{a}^{-}$, o conjunto de espécies eternas no tempo $t$ - digamos $\hat{\mathcal{X}}_{t}$ - restrito àquelas com aptidões acima de $a$ é dado por

$$
\mathcal{X}_{a}^{\prime} \cup \tilde{\mathcal{X}}_{a, t}
$$

onde $\mathcal{X}_{a}^{\prime}=\left.\mathcal{X}_{\mathcal{R}_{a}^{-}}\right|_{(a, 1]}$ e $\tilde{\mathcal{X}}_{a, t}=\left.\mathcal{N}\right|_{\left(\mathcal{R}_{a}^{-}, t\right) \times(a, 1]}$. Daí, para todo $t>\mathcal{R}_{a}^{-}$, para cada intervalo fechado $I \subset(a, 1]$, segue que

$$
\left.\left.\frac{1}{t}\left|\hat{\mathcal{X}}_{t}\right|_{I}\left|=\frac{1}{t}\right| \mathcal{X}_{a}^{\prime}\right|_{I}\left|+\frac{1}{t}\right| \tilde{\mathcal{X}}_{a, t}\right|_{I} \mid \text {. }
$$

Da finitude quase certa de $\mathcal{R}_{a}^{-}$e de $\mathcal{X}_{0}=B$, segue a finitude quase certa de $\left|\mathcal{X}_{a}^{\prime}\right|_{I} \mid$, o que garante que

$$
\frac{1}{t}\left|\mathcal{X}_{a}^{\prime}\right|_{I} \mid \rightarrow 0 \text {, quase certamente quando } t \nearrow \infty .
$$

Por outro lado, dos resultados acerca da Teoria da Renovação obtidos pela Lei Forte dos Grandes Números - ver (Durret, 2010), Capítulo 2, Teorema 2.4.6 - temos que

$$
\frac{1}{t}\left|\tilde{\mathcal{X}}_{a, t}\right|_{I}|\rightarrow(1-a)| I \mid, \text { quase certamente quando } t \nearrow \infty .
$$

\subsection{Processo pontual de surgimento de espécies eternas}

Partindo de um sistema desabitado, a cada instante de nascimento estará associada apenas uma espécie ou, de maneira equivalente, uma aptidão. Dentre todos estes instantes, há aqueles que são os de nascimentos de espécies eternas. Assim, podemos identificar cada surgimento de espécie eterna por um par ordenado $(x, y) \in(0, \infty) \times[0,1]$, onde $x$ indica o instante do nascimento e $y$ a respectiva aptidão associada. Isto é, o conjunto de espécies eternas pode ser representado por meio de pontos em $(0, \infty) \times[0,1]$; o que pode ser visto, para cada $\omega \in \Omega$, como uma realização de um processo pontual simples - vamos denotá-lo por $\hat{\mathcal{P}}-$ em $\mathbb{R}_{+} \times[0,1]$. Nessa seção, iremos discorrer sobre a função zero de $\hat{\mathcal{P}}$ e apresentamos estimativas para a taxa de decaimento (exponencial) da cauda da distribuição do tempo de surgimento da primeira espécie imortal a partir da condição inicial vazia.

De modo mais formal,

$$
\hat{\mathcal{P}}=\left\{\left(T_{n}, X_{n}\right) \in \mathcal{N}: X_{n} \text { representa uma espécie eterna }\right\} .
$$

Para todo boreliano $A \subset \mathcal{B}((0, \infty) \times[0,1])$, definamos

$$
\hat{N}(A)=|\hat{\mathcal{P}} \cap A|,
$$

que indica o número de espécies eternas surgidas em $A$. Agora, vamos introduzir a função zero do processo $\hat{\mathcal{P}}$, que é dada por

$$
\zeta(A)=\mathbb{P}(\hat{N}(A)=0) ;
$$

e indica a probabilidade de inexistência de nascimentos de espécies eternas em $A$. Vamos introduzir algumas variáveis auxilares para, a partir delas, apresentar uma maneira de caracterizar $\zeta(A)$ quando $A$ é dado por uma união finita de retângulos com medidas de Lebesgue finitas.

Para todo $A \subset \mathcal{B}((0, \infty) \times[0,1])$, consideramos os seguintes elementos: $x^{-}=\inf \{x:(x, y) \in A\}$, $x^{+}=\sup \{x:(x, y) \in A\}, y^{-}=\inf \{y:(x, y) \in A\}, y^{+}=\sup \{y:(x, y) \in A\}$. Note que $A \subset\left[x^{-}, x^{+}\right] \times\left[y^{-}, y^{+}\right]$. Ademais, para cada $y \in \operatorname{proj}_{y}(A):=\{y:(x, y) \in A\}$, consideramos 
$x_{y}^{-}=\inf \{x:(x, y) \in A\}, x_{y}^{+}=\sup \{x:(x, y) \in A\}, \mathrm{e}$

$$
A_{y}^{\downarrow}=A \cap\left[x^{-}, x^{+}\right] \times\left[y^{-}, y\right], \quad A_{y}^{\uparrow}=A \cap\left[x^{-}, x^{+}\right] \times\left(y, y^{+}\right] .
$$

É claro que $A_{y}^{\downarrow} \cap A_{y}^{\uparrow}=\emptyset$ e $A=A_{y}^{\downarrow} \cup A_{y}^{\uparrow}$. Agora, vamos introduzir

$$
X^{*}=\left(X_{1}^{*}, X_{2}^{*}\right) \in \mathcal{N} \cap A,
$$

onde $X_{2}^{*}=\max \left\{x_{2}: x=\left(x_{1}, x_{2}\right) \in \mathcal{N} \cap A\right\}$, com a convenção de que $\{\max \emptyset\}=y^{-}$. Note que $X_{1}^{*}$ depende de $X_{2}^{*}$. Para que $X_{1}^{*}$ esteja bem definida, façamos $X_{1}^{*}=x_{y^{-}}^{-}$sempre que $\mathcal{N} \cap A=\emptyset$. Resulta que,

$$
\begin{aligned}
\mathbb{P}\left(X_{2} \leq x_{2}\right) & =\mathbb{P}\left(X_{2} \leq x_{2} \mid \mathcal{N} \cap A=\emptyset\right) \mathbb{P}(\mathcal{N} \cap A=\emptyset)+\mathbb{P}\left(X_{2} \leq x_{2}, \mathcal{N} \cap A \neq \emptyset\right) \\
& =e^{-|A|}+\mathbb{P}\left(\mathcal{N} \cap A_{x_{2}}^{\downarrow} \neq \emptyset, \mathcal{N} \cap A_{x_{2}}^{\uparrow}=\emptyset\right) \\
& =e^{-|A|}+\left(1-e^{-\left|A_{x_{2}}^{\downarrow}\right|}\right) e^{-\left|A_{x_{2}}^{\uparrow}\right|}, \quad \text { para } x_{2} \in \operatorname{proj}_{y}(A),
\end{aligned}
$$

onde $|A|$ representa a medida de Lebesgue do conjunto $A$. A partir daqui, vamos supor que $A$ seja dado por uma união finita de retângulos com medidas de Lebesgue finitas e não-nulas. Decorre que

$$
\mathbb{P}\left(X_{2} \leq x_{2} \mid \mathcal{N} \cap A \neq \emptyset\right)=\frac{e^{-\left|A_{x_{2}}^{\uparrow}\right|}-e^{-\left|A_{x_{2}}^{\uparrow}\right|} e^{-\left|A_{x_{2}}^{\downarrow}\right|}}{1-e^{-|A|}}=\frac{e^{-\left|A_{x_{2}}^{\uparrow}\right|}-e^{-|A|}}{1-e^{-|A|}}, \text { para } x_{2} \in \operatorname{proj}_{y}(A) .
$$

Por um procedimento análogo, poderíamos obter a distribuição de $X_{1}^{*}$. Note que, pelas propriedades bem conhecidas dos Processos de Poisson, - vide (Ferrari e Galves, 1997), Lema 5.6, por exemplo dados $\mathcal{N} \cap A \neq \emptyset$ e $X_{2}^{*}=x_{2}^{*}, X_{1}^{*}$ é uniformemente distribuída em $\operatorname{proj}_{x}\left(A ; x_{2}^{*}\right):=\left\{x:\left(x, x_{2}^{*}\right) \in A\right\}$. Ademais, seja

$$
T=\left\{\begin{array}{lr}
\min \left\{t>X_{1}^{*}: M_{X_{2}^{*}, t}=0\right\}, & \text { se } \mathcal{N} \cap A \neq \emptyset \\
+\infty, & \text { caso contrário. }
\end{array}\right.
$$

Note que, dados $\mathcal{N} \cap A \neq \emptyset, X^{*}=\left(x_{1}^{*}, x_{2}^{*}\right)$ e $M_{x_{2}^{*}, x_{1}^{*}}, T-x_{1}^{*} \sim T^{x_{2}^{*}, M_{x_{2}^{*}, x_{1}^{*}+1,0}}$, isto é, $T-x_{1}$ consiste do tempo até a primeira passagem do processo $\left\{M_{x_{2}^{*}, t}: t \geq 0\right\}$ pelo estado zero a partir da condição inicial $M_{x_{2}^{*}, 0}=M_{x_{2}^{*}, x_{1}^{*}}+1$.

Proposição 3.2.1. A partir da condição inicial vazia, segue que

$$
\zeta(A)=e^{-|A|}+\mathbb{P}\left(\infty>T>x^{+}\right)+\int_{A} \int_{x_{1}^{*}}^{x^{+}} f_{T}(s) \zeta\left(A_{s}^{*}\right) f_{X^{*}}\left(x^{*}\right) d s d x^{*},
$$

onde $A_{t}^{*}=A \cap[t, \infty) \times\left[0, x_{2}^{*}\right]$.

Demonstração. Basta ver que

$$
\zeta(A)=\mathbb{P}(\mathcal{N} \cap A=\emptyset)+\mathbb{P}(\hat{N}(A)=0, \mathcal{N} \cap A \neq \emptyset)=e^{-|A|}+\mathbb{P}(\hat{N}(A)=0, \mathcal{N} \cap A \neq \emptyset),
$$

e que

$$
\begin{aligned}
\mathbb{P}(\hat{N}(A)=0, \mathcal{N} \cap A \neq \emptyset)= & \mathbb{P}\left(\hat{N}(A)=0, \mathcal{N} \cap A \neq \emptyset, T>x^{+}\right) \\
& +\mathbb{P}\left(\hat{N}(A)=0, \mathcal{N} \cap A \neq \emptyset, T \leq x^{+}\right) \\
= & \mathbb{P}\left(\infty>T>x^{+}\right)+\int_{A} \int_{x_{1}^{*}}^{x^{+}} f_{T}(s) \zeta\left(A_{s}^{*}\right) f_{X^{*}}\left(x^{*}\right) d s d x^{*},
\end{aligned}
$$

onde $A_{t}^{*}=A \cap[t, \infty) \times\left[0, x_{2}^{*}\right]$. 
A equação integral 3.17 parece muito complicada para ser resolvida mesmo para o caso mais simples em que $A$ é um retângulo. Vamos a seguir obter uma cota inferior para $\zeta(A)$ no caso em que $A=[0, t] \times[0,1]$, o que nos dá uma cota inferior para a cauda da distribuição do tempo de surgimento da primeira espécie eterna. Em segundo momento, por um argumento independente, obtemos uma cota superior para a mesma cauda.

\section{Cota inferior para a cauda da distribuição do tempo de surgimento da primeira espécie eterna}

Seja $\mathscr{T}$ o tempo até o aparecimento da primeira espécie eterna partindo da condição inicial vazia. Ademais, dados $0<s<t$ e $0<u<1$, sejam $B:=[0, t] \times[0,1], \tilde{\zeta}(s, u):=\zeta(A)$, onde $A=$ $[s, t] \times[0, u]$ e $\tilde{\zeta}(t):=\tilde{\zeta}(0,1)=\zeta(B)$. Não deve ser difícil ver que os eventos $\{\mathscr{T}>t\}$ e $\{\hat{\mathcal{P}} \cap B=\emptyset\}$ são equivalentes. Logo,

$$
\mathbb{P}(\mathscr{T}>t)=\tilde{\zeta}(t)
$$

Pela Proposição 3.2.1, temos

$$
\tilde{\zeta}(t)=e^{-t}+\mathbb{P}(\infty>T>t)+\int_{B} \int_{x_{1}^{*}}^{t} f_{T}(s) \tilde{\zeta}\left(s, x_{2}^{*}\right) f_{X^{*}}\left(x^{*}\right) d s d x^{*} .
$$

Das equações 3.18 e 3.19 , segue que $\mathbb{P}(\mathscr{T}>t) \geq e^{-t}$. Vamos refinar essa desigualdade. Como observado na seção anterior, todas as espécies com aptidões menores que $\mu$ desaparecem quase certamente depois de um tempo finito, isto é, $\zeta(A)=1$ para todo $A \subset \mathcal{B}((0, \infty) \times[0, \mu))$. Daí, para todo $A \subset \mathcal{B}((0, \infty) \times[0,1])$,

$$
\zeta(A)=\mathbb{P}\left(\hat{N}\left(A_{\mu}^{\uparrow}\right)=0\right) \mathbb{P}\left(\hat{N}\left(A_{\mu}^{\downarrow}\right)=0 \mid \hat{N}\left(A_{\mu}^{\uparrow}\right)=0\right)=\mathbb{P}\left(\hat{N}\left(A_{\mu}^{\uparrow}\right)=0\right)=\zeta\left(A_{\mu}^{\uparrow}\right) .
$$

A partir de $\zeta(A)=\zeta\left(A_{\mu}^{\uparrow}\right)$, podemos reescrever a equação integral 3.19, que fica dada por

$$
\tilde{\zeta}(t)=e^{-(1-\mu) t}+\mathbb{P}(\infty>T>t)+\int_{B_{\mu}^{\uparrow}} \int_{x_{1}^{*}}^{t} f_{T}(s) \tilde{\zeta}\left(s, x_{2}^{*}\right) f_{X^{*}}\left(x^{*}\right) d s d x^{*} .
$$

Logo,

$$
\mathbb{P}(\mathscr{T}>t) \geq e^{-(1-\mu) t}
$$

Da desigualdade 3.22 , segue que

$$
\liminf _{t \nearrow \infty} \frac{1}{t} \log \mathbb{P}(\mathscr{T}>t) \geq-(1-\mu) .
$$

Para a obtenção da cota inferior dada acima utilizamos o primeiro termo de 3.21. Observamos que a obtenção do comportamento do segundo termo de 3.21 parece factível, o que possivelmente melhore essa cota pelo menos em alguns casos. A seguir, vamos mostrar que $\mathbb{P}(\mathscr{T}>t)$ é cotada superiormente por uma função que possuí decaimento exponencial em $t$.

Cota superior para a cauda da distribuição do tempo de surgimento da primeira espécie eterna

Na demonstração do Teorema 3.1.1, para cada $a \in(\mu, 1]$, denotamos por $\mathcal{S}_{a}$ o tempo até o surgimento de uma espécie eterna com aptidão acima de $a$. Note que, para cada $a \in(\mu, 1], \mathscr{T} \preceq \mathcal{S}_{a}$. 
Daí,

$$
\mathbb{P}(\mathscr{T}>t) \leq \mathbb{P}\left(\mathcal{S}_{a}>t\right), \quad \text { para todo } a \in(\mu, 1] .
$$

Vamos obter a cota superior de interesse a partir da inequação acima. Para isso, recapitulamos que

$$
\mathcal{S}_{a}=\tau_{a}+\phi_{a_{\downarrow}}+\phi_{a_{\uparrow}}
$$

onde $\phi_{a_{\downarrow}} \sim \exp (a)$ e $\phi_{a_{\uparrow}} \sim \exp (1-a)$ e $\tau_{a}$ são independentes, e

$$
\tau_{a}=\sum_{k=1}^{N_{a}} T_{a, k},
$$

com $N_{a}$ representando o número de retornos de $\left\{M_{a, t}^{0}: t \geq 0\right\}$ à origem e, pela propriedade de Markov, $T_{a, 1}, \ldots, T_{a, N_{a}}$ são i.i.d. e independentes de $N_{a}$ tal que

$$
T_{a, 1} \stackrel{\mathscr{P}}{=} \sum_{k=1}^{L_{a}} \xi_{a, k},
$$

onde $L_{a}$ é o tempo de retorno, dado que é finito, de $\left\{L_{a, n}: n \geq 0\right\}$ à origem e $\xi_{a, 1}, \ldots, \xi_{a, L_{a}}$ são variáveis aleatórias i.i.d. e independentes de $L_{a}$, $\operatorname{com} \xi_{a, 1} \sim \exp (a+\mu)$.

Seja $K$ o tempo de retorno de $\left\{L_{a, n}: n \geq 0\right\}$ à origem. Note que $L_{a}=K \mid K<\infty$ e que $\rho=\mathbb{P}(K=$ $\infty)$. Dos resultados bem conhecidos sobre o tempo de retorno de um passeio aleatório simples à origem - vide (Grinstead e Snell, 2003), capítulo 12, seção 2 - segue que $\rho=\mu / a$ e que

$$
\mathbb{E}\left(s^{L_{a}}\right)=\frac{a+\mu-\sqrt{(a+\mu)^{2}-4 a \mu s^{2}}}{2 \mu}, \quad \text { para } s \leq \frac{a+\mu}{2 \sqrt{a \mu}} .
$$

Ademais, é bem sabido que

$$
\mathbb{E}\left(s^{\xi_{a, 1}}\right)=\frac{a+\mu}{a+\mu-\log s}, \quad \text { para } \log s<a+\mu,
$$

e que

$$
\mathbb{E}\left(s^{N_{a}}\right)=\frac{\rho}{1-(1-\rho) s}=\frac{a-\mu}{a+\mu s}, \quad \text { para } s<\frac{a}{\mu} .
$$

Segue que

$$
\begin{aligned}
\mathbb{E}\left(s^{T_{a, 1}}\right) & =\left.\mathbb{E}\left(t^{L_{a}}\right)\right|_{t=\mathbb{E}\left(s^{\xi_{a, 1}}\right)} \\
& =\frac{(a+\mu)(a+\mu-\log s)-(a+\mu) \sqrt{(a+\mu-\log s)^{2}-4 a \mu}}{2 \mu(a+\mu-\log s)},
\end{aligned}
$$

para $\log s \leq a+\mu-2 \sqrt{a \mu}$, e $\operatorname{logo}$ que

$$
\begin{aligned}
\mathbb{E}\left(s^{\tau_{a}}\right) & =\left.\mathbb{E}\left(u^{N_{a}}\right)\right|_{u=\mathbb{E}\left(s^{T_{a, 1}}\right)} \\
& =\frac{2(a-\mu)(a+\mu-\log s)}{(a-\mu)(a+\mu-\log s)+(a+\mu) \sqrt{(a+\mu-\log s)^{2}-4 a \mu}},
\end{aligned}
$$

para $\log s \leq a+\mu-2 \sqrt{a \mu}$. Portanto,

$$
\begin{aligned}
\mathbb{E}\left(s^{\mathcal{S}_{a}}\right) & =\frac{a}{a-\log s} \times \frac{1-a}{1-a-\log s} \times \\
& \times \frac{2(a-\mu)(a+\mu-\log s)}{(a-\mu)(a+\mu-\log s)+(a+\mu) \sqrt{(a+\mu-\log s)^{2}-4 a \mu}},
\end{aligned}
$$


para $\log s \leq a+\mu-2 \sqrt{a \mu}$ e $\log s<1-a$. De modo geral, $\mathbb{E}\left(s^{\mathcal{S}_{a}}\right)$ está bem definida e é dada como acima em $U \subset\{(a, s): a \in(\mu, 1]$ e $s \in \mathbb{R}\}$ determinado pelas condições log $s \leq a+\mu-2 \sqrt{a \mu}$ - crescente em $a$ para $a \geq \mu$ - se $\mu<a<1 / 2+\sqrt{2 \mu-\mu^{2}} / 2$ e $\log s<1-a$ - decrescente em $a$ - se $1 / 2+\sqrt{2 \mu-\mu^{2}} / 2 \leq a<1$.

Pela desigualdade de Markov, segue que

$$
\mathbb{P}(\mathscr{T}>t) \leq \mathbb{P}\left(\mathcal{S}_{a}>t\right) \leq s^{-t} \mathbb{E}\left(s^{\mathcal{S}_{a}}\right), \text { para todo }(a, s) \in U,
$$

e concluímos que

$$
\limsup _{t \nearrow \infty} \frac{1}{t} \log \mathbb{P}(\mathscr{T}>t) \leq-\log s \leq-\min \{a+\mu-2 \sqrt{a \mu}, 1-a\}, \text { para todo }(a, s) \in U .
$$

Segue que

$$
\begin{aligned}
\limsup _{t \operatorname{su}_{\infty}} \frac{1}{t} \log \mathbb{P}(\mathscr{T}>t) & \leq-\sup _{(a, s) \in U} \min \{a+\mu-2 \sqrt{a \mu}, 1-a\} \\
& =-\max _{a \in(\mu, 1]} \min \{a+\mu-2 \sqrt{a \mu}, 1-a\} \\
& =-\frac{1-\sqrt{2 \mu-\mu^{2}}}{2} .
\end{aligned}
$$

Juntando as cotas em 3.23 e 3.33 , obtemos o seguinte resultado.

Proposição 3.2.2. Dado $0<\mu<1$, temos que

$$
-(1-\mu) \leq \liminf _{t \nearrow \infty} \frac{1}{t} \log \mathbb{P}(\mathscr{T}>t) \leq \limsup _{t \nearrow \infty} \frac{1}{t} \log \mathbb{P}(\mathscr{T}>t) \leq-\frac{1-\sqrt{2 \mu-\mu^{2}}}{2} .
$$




\section{Capítulo 4}

\section{Conclusões}

Nessa dissertação, a partir de um caso particular do modelo GMS, discorremos sobre duas questões inerentes ao modelo: a distribuição assintótica das espécies na fase subcrítica do processo e o eventual surgimento de espécies que sobrevivem para sempre. Os resultados, apesar de obtidos para um caso particular, podem ser estendidos/reencontrados para o caso geral, uma vez que existe uma relação direta entre o caso geral e o caso particular considerado. 
CONCLUSÕES 


\section{Referências Bibliográficas}

Billingsley (1968) Patrick Billingsley. Convergence of probability measures. John Wiley \& Sons, primeira ed. Citado na pág. 10, 11

Daley e Vere-Jones (2008) D. J. Daley e D. Vere-Jones. An introduction to theory of point process: general theory and structure, volume II. Springer, segunda ed. Citado na pág. 4

Durret (2010) Richard Durret. Probability. Theory and Examples. Cambridge University Press, quarta ed. Citado na pág. 19

Durret (2012) Richard Durret. Essentials of Stochastic Process. Springer, segunda ed. Citado na pág. 6

Elaydi (2005) Saber Elaydi. An introduction to difference equations. Springer, terceira ed. Citado na pág. 8

Ethier e Kurtz (1986) Stewart N. Ethier e Thomas G. Kurtz. Markov processes. Caracterization and convergence. John Wiley \& Sons, primeira ed. Citado na pág. 10

Ferrari e Galves (1997) Pablo A. Ferrari e Antonio Galves. Acoplamento e Processos Estocásticos. IMPA. Notas de aula. Citado na pág. 20

Grimmett e Stirzaker (2001) Geoffrey Grimmett e David Stirzaker. Probability and Random Process. Oxford, terceira ed. Citado na pág. 17

Grinstead e Snell (2003) Charles M. Grinstead e J. Laurie Snell. Introduction to Probability. American Mathematical Society, segunda ed. Citado na pág. 22

Guiol et al. (2011) Hervé Guiol, Fábio P. Machado e Rinaldo B. Schinazi. A stochastic model of evolution. Markov Process and Related Fields, 17:253-258. Citado na pág. 1

Guiol et al. (2013) Hervé Guiol, Fábio P. Machado e Rinaldo B. Schinazi. On a link between a species survival time in a evolution model and the bessel distributions. Brazilian Journal of Probability and Statistics, 27:201-209. Citado na pág. 1, 4, 17

Khouri (2013) Renata Stella Khouri. Estudos sobre um modelo estocástico para a evolução de uma espécie. Dissertação de Mestrado, Instituto de Matemática e Estatística, Universidade de São Paulo, Brasil. Citado na pág. 1

Preston (1975) Chris Preston. Spatial birth-and-death processes. Bull. Inst. Internat. Statist, 46:371-391. Citado na pág. 14 